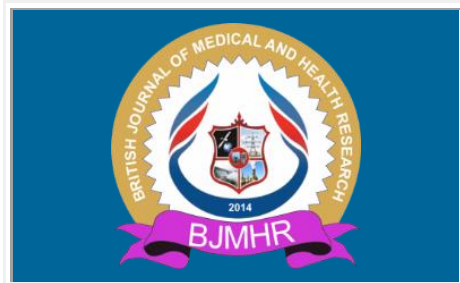

\title{
BJMHR
}

British Journal of Medical and Health Research

Journal home page: www.bjmhr.com

\section{Knowledge of the Glasgow Coma Scale among Nurses in Masaba North Sub-County Hospital}

\author{
Kariuki Pauline Njoki ${ }^{1 *}$, Omariba Motari ${ }^{2}$ \\ 1.Department of Nursing. Masaba North Sub-County Hospital, P.O. Box 228-40202, \\ Keroka, Kenya.
}

2. Department of Physiotherapy, Kenya Medical Training College (KMTC), Homa Bay Campus P/O.Box 40300-512 Homa Bay, Kenya.

\begin{abstract}
The Glasgow Coma Scale is a tool used to aid in objectively measuring the neurological status of a patient. The objectives of the study were to (i) examine the relationship of the demographics of the nurses and their knowledge of the Glasgow Coma Scale; (ii) find out about exposure of the nurses to the Glasgow Coma Scale; and (iii) assess their knowledge of Glasgow Coma Scale. The study was conducted in Masaba North Sub-County Hospital, Nyamira County, Kenya. The study population was all the nurses working in that hospital. The eligibility criteria were all nurses working during the data collection period and the exclusion criteria was all the nurses on study leave, maternity leave, and sick leave. The study design was crosssectional using a quantitative approach to data collection. Data were collected using a structured self-administered questionnaire. The study concluded that: (i) there was low level of knowledge about Glasgow Coma Scale; and (ii) there was inappropriate application of basic theoretical knowledge to clinical scenarios. It is therefore recommended that: (i) the length and depth of both the teaching of the theoretical concepts and the skill of performing Glasgow Coma Scale assessment be reviewed by nursing schools for basic and post-basic educational programmes for nursing students with the view of adapting more comprehensive in-depth teaching combined with demonstrations in classrooms and during clinical experiences in order to enhance nurses' knowledge of the Tool; and (ii) implementation of regular refresher training sessions.
\end{abstract}

Key words: Kenya, knowledge, nurses, Glasgow Coma Scale 


\section{INTRODUCTION}

The Glasgow Coma Scale (GCS) is a tool that is employed worldwide to identify neurologic dysfunction and follow-up progress of level of consciousness, predict prognosis, and standardize communication among health care professionals ${ }^{1}$.

The Scale was developed in 1974 by Graham Teasdale and Bryan J. Jennett, professors of neurosurgery at the University of Glasgow's Institute of Neurological Sciences in Scotland, UK. It is a tool used to aid in objectively measuring the neurological status of a patient. The tool has the ability to communicate the level of consciousness of patients. It is used to objectively measure coma severity in all acute medical and trauma patients. The Scale is the gold standard used for all acute medical and trauma patients. Although most commonly used in the Intensive Care Unit and Emergency Room setting, nurses may need to perform a GCS on a patient at any given time. Thus, it is a tool that requires the nurses to fully understand its purpose and how to use $\mathrm{it}^{2-5}$.

It was initially used to assist patients who suffered trauma, mainly traumatic brain injury victims, and, posteriorly, its use extended to other neurologic conditions that can alter consciousness. Its appeal lies in its applicability in a wide variety of clinical situations as well as its ease of use by a range of healthcare staff. It is an objective and reliable tool that nurses and nursing students (and indeed all health care workers) should become familiar with regardless of their place of employment ${ }^{2-5}$.

The Scale was designed as a way to communicate about the level of consciousness of patients with an acute brain injury. The findings using the scale guided initial decision making and monitored trends in responsiveness that were important in signaling the need for new actions ${ }^{5}$. There are three aspects of behaviour that are measured as part of an assessment of a patient's GCS: eye-opening, verbal performance and motor responsiveness. Each component is assessed independently and given a score. The maximum possible score for opening of the eyes is 4 points. The maximum possible score for verbal response is 5 points and the maximum possible score for motor response is 6 points. The highest response from each category elicited by the healthcare professional is scored on the chart. The final score is determined by adding the values for the three components and their sum is the GCS score. The highest possible score is $\mathbf{1 5}$ while the lowest possible score is $\mathbf{3}$. Once a score has been identified, it's important to understand its meaning. It is important to note that every brain injury is different, depending on the cause. But generally, brain injury is classified as: GCS of 3-8 as severe, GCS of 9-12 as moderate, and GCS of 13-15 as mild ${ }^{2-7}$.

The GCS allows healthcare professionals to consistently evaluate the consciousness level of a patient. It is commonly used in the context of head trauma, but it is also useful in a wide variety 
of other non-trauma related settings. By regularly assessing a patient's GCS scores, a downward trend in consciousness level can be recognized early, allowing time for appropriate interventions to be performed ${ }^{6}$.

The use of GCS requires previous knowledge and skills. This scale, applied carefully and systematically, is fundamental for assessment and establishment measures of the patient in order to guarantee reliability, which is critical to follow-up the progress of such patients. The results of the score are used to establish a baseline, then used to assess whether the patient's condition is improving, stable or deteriorating. The GCS is always used in conjunction with a patient history as many conditions and events may result in an abnormal score ${ }^{7}$.

Nurses need to acquire a wide range of theoretical and practical knowledge in order to provide the appropriate level of care for patients because they are responsible to continuously assess patients. The most important assessment of neurological examination in the clinical setting is assessing level of consciousness. Rapid and correct assessment will minimize the neurological complications, unnecessary and incorrect diagnostic procedures, mortality and morbidity. Knowledge of the GCS is thus recognized as an asset to all clinical nurses. Nurses not only need to know how to assess level of consciousness by GCS, but also they need to know how to interpret these numerical values.

However, many studies in different countries have reported low levels of knowledge of the GCS among nurses. For example, Summers and $\mathrm{McLeod}^{8}$ critically reviewed seven studies that addressed factors influencing nurses' performance of the GCS. Their review found that there were inconsistencies regarding the application of painful stimuli and accuracy of documentation. Another study noted that there was a lack of interest in the use of neurological assessments by nurses 9 .

Given the importance of GCS as a tool for neurological assessment of patients, and the need for careful and standardized application, this study was very significant because it laid the foundation for building a body of knowledge in this important area not just in Masaba North Sub-County Hospital, but in Nyamira County, in Kenya, in Africa, and all over the world. The study provided evidence-based data for future policies designed to improve knowledge about GCS. It also provided policy makers and program administrators with a better understanding of reasons why more effort should be put in achieving better knowledge about GCS for all nurses (and indeed health care professionals) all over the county, the nation, the continent, and all over the world.

\section{Objectives}

1. To examine relationship of the demographics of the nurses working in Masaba North SubCounty Hospital and their knowledge of the Glasgow Coma Scale.

2. To find out about exposure of the nurses to the Glasgow Coma Scale. 
3. To assess knowledge level of Glasgow Coma Scale among nurses.

\section{MATERIALS AND METHOD}

\section{Study site}

The study was conducted in Masaba North Sub-County Hospital, Nyamira County, Kenya. The hospital is situated in Keroka town along the Keroka-Nyangusu Road. It serves as the referral hospital for the sub-county. It is an out-patient as well as an in-patient facility. It has a bed capacity of 50 patients. It receives cases of traumatic brain injuries and other complicated forms of trauma and medical conditions in the sub-county, either as direct admissions or through referrals. Some of the patients require monitoring with the GCS.

\section{Study population}

The study population was all the nurses in Masaba North Sub-County hospital. The study employed saturated sampling method whereby the entire population of 35 nurses working in the different departments of the hospital were targeted to participate in the survey. However, the eligibility criteria were all nurses working during the data collection period and the exclusion criteria was all the nurses on study leave, maternity leave, and sick leave.

\section{Study design}

The study design was cross-sectional, which used a quantitative approach to data collection to determine the level of knowledge of Glasgow Coma Scale among nurses in Masaba North SubCounty hospital.

\section{Data collection instrument}

The instrument for data collection was a structured self-administered questionnaire developed by the researchers according to the specific objectives. The tool comprised of four sections. Section 1 had five questions and captured data on the demographic characteristics of the respondents, mainly, gender, age, level of nursing education, years of experience and the department she/he was currently working in. Section 2 also had five questions and captured data on exposure to GCS, mainly, whether the respondent was taught GCS in nursing school, whether she/he had ever performed GCS assessment in the clinical area, whether she/he had ever seen the GCS chart, whether she/he had ever used the GCS chart, and finally, whether she/he had ever received any refresher training on the GCS. Section 3 had five multiple choice questions that tested theoretical knowledge about the GCS, mainly, what type of tool it is, the best GCS score, the worst score, the critical score, and the score for moderate severity. The last section had fifteen multiple choice questions that assessed practical application of knowledge about the GCS in clinical scenarios by grading the different parameters of the GCS. 
In order to ensure validity and reliability of the questionnaire, it was pretested on a sample of 4 nurses working in a different sub-county hospital. No ambiguities were found in the questions, so it was maintained in its original form.

\section{Data collection procedure}

Following ethical approval from the Medical Superintendent of the Masaba Sub-County Hospital on 13/11/2019, data were then collected from December 2019 to February 2020. The researchers approached each nurse individually during their break times. They were given the approval letter to read which introduced the study and its purpose and explained to them the rights of the participants. Those who consented to participate in the study signed the consent form and were given the questionnaire and supervised to complete it within 30 minutes in the presence of the researcher so that they could not search for information which could have affected the validity of the results. Each questionnaire was checked for completeness immediately a participant finished answering. Those who did not complete any relevant portions were prompted to complete it. The questionnaire was then added to other questionnaires to maintain anonymity of the respondents.

\section{Data analysis, presentation and storage}

The Statistical Package for Social Sciences, version 22, was used to code, enter, analyse, organize, present and store data from the study. Descriptive statistics such as frequencies, percentages, and means were used to summarize the findings from the study. Knowledge and application of knowledge about the GCS was determined from a scale of 20 multiple choice questions. Each correct answer was valued at five points, and a wrong answer attracted no point. Questions that were not answered were treated as wrong answers. A total score was calculated for each respondent from these 20 multiple choice items. The respondents were then grouped into three categories based on their total score: good knowledge (above 80 percent), moderate knowledge (60 to 80 percent), and poor knowledge (less than 60 percent). Pearson correlations were performed to see if age, level of nursing education and years of experience in nursing have an association with knowledge. Data was presented using tables.

\section{Ethical considerations}

The study was approved by the Medical Superintendent of the Masaba North Sub-County Hospital. All the respondents signed the consent form. The identity of the respondents remained anonymous throughout the study and the data was kept confidential. Participation was voluntary for all the respondents. The researchers explained in detail the purpose of the study, content of the questionnaire, informed the respondents on confidentiality of their responses and of their free choice to withdraw from the study at any point. The identity of respondents remained anonymous throughout the study and the data kept confidential. Research results 
were reported accurately and honestly and the study findings communicated to all the interested parties.

\section{Limitations for the study}

The main limitations of this study were: (i) it was conducted in a single health facility, therefore, it constitutes a local experience; and (ii) a small population size was used which limits generalization of the findings.

\section{RESULTS AND DISCUSSION}

\section{Demographic characteristics}

Out of a total targeted population of 35 nurses, 29 nurses $(82.9 \%)$ participated in the study. Majority $72.41 \%$ were females while $27.59 \%$ were males. The demographic characteristics of the respondents are shown in table 1 below.

Table 1: Demographic characteristics of the respondents $(n=29)$

\begin{tabular}{|c|c|c|}
\hline Characteristics & Frequency & Percentage \\
\hline \multicolumn{3}{|l|}{ Gender } \\
\hline Male & 8 & 27.59 \\
\hline Female & 21 & 72.41 \\
\hline Total respondents & 29 & 100.00 \\
\hline \multicolumn{3}{|l|}{ Age } \\
\hline $18-30$ years & 2 & 06.90 \\
\hline $31-40$ years & 17 & 58.62 \\
\hline $41-50$ years & 6 & 20.69 \\
\hline $51-60$ years & 4 & 13.79 \\
\hline \multicolumn{3}{|l|}{ Level of nursing education } \\
\hline Certificate & 4 & 13.79 \\
\hline Diploma & 18 & 62.07 \\
\hline Degree & 7 & 24.14 \\
\hline Masters & 0 & 0 \\
\hline $\mathrm{PhD}$ & 0 & 0 \\
\hline \multicolumn{3}{|l|}{ Years of nursing experience } \\
\hline $0-5$ & 1 & 03.45 \\
\hline $6-10$ & 11 & 37.93 \\
\hline $11-15$ & 7 & 24.14 \\
\hline $16-20$ & 3 & 10.34 \\
\hline $21-25$ & 3 & 10.34 \\
\hline $26-30$ & 4 & 13.80 \\
\hline 30 and above & 0 & 0 \\
\hline \multicolumn{3}{|l|}{ Department } \\
\hline Nursing office & 1 & 03.45 \\
\hline Maternal and Child Health clinic & 4 & 13.80 \\
\hline Comprehensive Care Centre & 2 & 06.90 \\
\hline Maternity & 9 & 31.02 \\
\hline Out-patient Department & 7 & 24.14 \\
\hline Wards & 6 & 20.69 \\
\hline
\end{tabular}

Exposure to the GCS 
All except one respondent had been taught GCS in nursing school, while only one respondent had received refresher training on the GCS. Results on exposure to GCS are shown in table 2 below.

Table 2: Exposure to the Glasgow Coma Scale $(n=29)$

\begin{tabular}{lll}
\hline & Yes & No \\
\hline Were you taught about GCS in nursing school? & 28 & 1 \\
Have you ever performed GCS assessment in the clinical area? & 21 & 8 \\
Have you ever seen the GCS chart? & 23 & 6 \\
Have you ever used the GCS chart? & 17 & 12 \\
Have you ever received any refresher training on the GCS? & 1 & 28 \\
\hline
\end{tabular}

\section{Theoretical knowledge about GCS}

Theoretical knowledge of the GCS was assessed using five multiple choice questions, each five points. The overall average score for the five questions was 16.9 out of 25 (67.6\%). The summary of the results are shown in table 3 below.

\section{Practical application of knowledge about the GCS}

Practical application of knowledge about the GCS was assessed using fifteen multiple choice questions, each five points. The overall average score for the fifteen questions was 37.76 out of $75(50.35 \%)$.

The summary of the results are shown in table 3 below.

\section{Knowledge of GCS}

Knowledge on GCS was assessed through the addition of the theoretical knowledge scores and the scores for the application of knowledge about GCS as shown in the last column on the right of table 3 below.

Results show that the overall score for knowledge on GCS was 54.46\%. More than half (55.17 $\%$ ) of the respondents performed poorly in the study, scoring below $60 \% .31 .04 \%$ had average scores of between $60-80 \%$, while only $13.79 \%$ scored above $80 \%$.

Table 3: Knowledge of GCS scores $(\mathbf{n}=29)$

\begin{tabular}{llll}
\hline Characteristics & Theory & Application & Total score (\%) \\
\hline Gender & & & \\
Male & 18.13 & 38.13 & 56.26 \\
Female & 16.43 & 37.62 & 54.05 \\
Averages & $\mathbf{1 6 . 9 0}$ & $\mathbf{3 7 . 7 6}$ & $\mathbf{5 4 . 6 6}$ \\
Age & & & \\
18-30 years & 20.00 & 40.00 & 60.00 \\
31-40 years & 17.06 & 38.53 & 55.59 \\
41-50 years & 14.17 & 32.50 & 46.67 \\
51-60 years & 17.50 & 41.25 & 58.75 \\
Level of nursing education & & & \\
Certificate & 13.75 & 37.50 & 51.25 \\
Diploma & 15.00 & 34.44 & 49.44 \\
Degree & 22.86 & 46.43 & 69.29 \\
Masters & 0 & 0 & 0 \\
\hline
\end{tabular}




\begin{tabular}{llll}
\hline PhD & 0 & 0 & 0 \\
Years of nursing experience & & & \\
$0-5$ & 25.00 & 60.00 & 85.00 \\
$6-10$ & 15.91 & 34.55 & 50.46 \\
$11-15$ & 16.43 & 35.71 & 52.14 \\
$16-20$ & 16.67 & 35.00 & 51.67 \\
$21-25$ & 20.00 & 46.67 & 66.67 \\
$26-30$ & 15.00 & 40.00 & 55.00 \\
30 and above & 0 & 0 & 0 \\
Department & & & \\
Nursing office & 20.00 & 40.00 & 60.00 \\
Maternal and Child Health clinic & 13.75 & 36.25 & 50.00 \\
Comprehensive Care Centre & 22.50 & 60.00 & 82.50 \\
Maternity & 13.89 & 37.22 & 51.11 \\
Out-patient Department & 18.57 & 32.14 & 50.71 \\
Wards & 18.33 & 38.33 & 56.66 \\
\hline
\end{tabular}

\section{Bivariate correlation of knowledge of GCS and selected demographic characteristics}

Gender, age, level of nursing education and years of nursing experience were correlated with knowledge of GCS. Only the level of nursing education had a positive correlation. The results are shown in table 4 below.

Table 4: Correlation of demographic characteristics with knowledge on GCS

\begin{tabular}{|c|c|c|c|c|}
\hline \multirow[t]{2}{*}{ Variable } & \multicolumn{4}{|c|}{$P$ values for knowledge on GCS } \\
\hline & $p=99 \% C I$ & $p=95 \% C I$ & $r=99 \% C I$ & Sig. (2-tailed) \\
\hline Gender & & \multirow{4}{*}{0.413} & 0.015 & 0.937 \\
\hline Age & -0.179 & & & 0.352 \\
\hline Level of nursing education & & & & 0.026 \\
\hline Years of nursing experience & -0.119 & & & 0.538 \\
\hline
\end{tabular}

\section{DISCUSSION}

This study assessed knowledge of the Glasgow Coma Scale among nurses working in a subcounty hospital in Kenya. Overall results for the study showed that the total average score was $54.46 \%$, revealing that the nurses had poor knowledge of the GCS. Unfortunately, many studies that have been conducted to assess knowledge of nurses about the GCS have reported similar results showing that nurses generally have poor knowledge about this important tool ${ }^{1,6,8-}$ 26.

\section{Demographic characteristics}

\section{Gender}

Male nurses accounted for $27.59 \%$ while the female nurses accounted for $72.41 \%$. However, results showed that the male gender had slightly higher levels of knowledge about GCS (55.63 $\%)$ as compared with females $(54.05 \%)$. One study ${ }^{16}$ found that female nurses demonstrated significantly higher levels of knowledge than male nurses contradicting with the result of the current study. 
Nonetheless, Spearman's correlation of gender with knowledge of GCS was insignificant $(r=0.015)$, showing that there was no correlation between the two. One study ${ }^{15}$ found that there were no significant associations between nurses' gender and knowledge of GCS concurring with the results of the current study. However, another study ${ }^{12}$ found that there was a significant relationship between gender and level of GCS knowledge, contradicting with the results.

\section{Age}

The respondents of this study were relatively young with more than half of them $(58.62 \%)$ being in their thirties. In one study ${ }^{1}$, the mean age was 31.1 years while another study ${ }^{16}$ found that most of the respondents were in their thirties as well. This shows a relatively young generation of nurses, who are expected to be very knowledgeable. But results showed that this age group (31-40) scored $55.59 \%$ while the oldest age group (51-60 years) scored more than them $(58.75 \%)$.

However, Pearson's correlation revealed that age was negatively correlated, although weakly, with knowledge of GCS ( $p=-0.179)$. One study ${ }^{15}$ found that there were no significant associations between nurses' age and GCS knowledge. However, two studies found that age was positively correlated with knowledge of GCS, contradicting with the current study ${ }^{16,17}$.

\section{Level of nursing education}

The majority of the respondents had a Diploma in nursing (62.07\%). Pearson's correlation of knowledge of GCS with level of nursing education was positive ( $p=0.413)$, showing that there was a positive correlation of level of education with knowledge of GCS. Some studies found similar results ${ }^{17,22-24}$. However, other studies ${ }^{13-16,20}$ found that there was no relationship between the level of education and knowledge of GCS, contradicting with the current study.

\section{Years of nursing experience}

In this study, the Pearson's correlation of years of nursing experience with knowledge of GCS was weakly negative $(p=-0.119)$, showing there is no relationship between experience as a nurse and knowledge of GCS. Other studies ${ }^{13,15-17}$ have found similar results concurring with the results. However, one study ${ }^{1}$ found that nurses who had graduated more than 5 years ago presented a lower percentage success rate, and that the more time of experience, the higher was the percentage of right answers, showing a positive correlation of experience and GCS knowledge, contradicting with the results of the current study. Another study found that knowledge and experience correlated with accurate assessment of the Glasgow Coma Scale. However, scores between them were inaccurate ${ }^{8}$.

\section{Department}

The respondents working in the wards, which is where most patients who need monitoring using GCS are admitted and nursed, scored $56.66 \%$. This score was lower than that for the Comprehensive Care Centre $(82.5 \%)$ and the nursing office $(60 \%)$. This can be explained by 
the fact that all the nurses in the Comprehensive Care Centre and the nursing office are graduates and correlation results revealed that the level of nursing education was positively correlated with knowledge of GCS.

One study found that nurses from emergency service had more correct answers than nurses from intensive care unit ${ }^{1}$. Another study found that Emergency Room nurses recorded less level of knowledge than other areas of practice ${ }^{13}$. In another study, significant differences were found in the GCS knowledge of the nurses across the various wards/units, with neurological ward nurses having the highest score ${ }^{15}$, while a different study found that knowledge of nurses working in the Neurosurgical ward about the GCS was statistically significantly higher because they frequently managed patients requiring monitoring with the $\mathrm{GCS}^{16}$.

\section{Exposure to GCS}

All except one respondent had been taught GCS in nursing school, while only one respondent among the respondents had received refresher training on the GCS. One study found that the number of years since graduation, experience, and work at critical care units interfered in nurses' knowledge about the scale, which indicates the need for refresher training ${ }^{1}$.

One study found that $5.1 \%$ of the respondents lacked basic training on GCS, while $47.6 \%$ of them had never attended any type of refresher training on $\mathrm{GCS}^{14}$. Another study found that even though $93 \%$ of the participants were taught about the GCS during the course of their training, the teaching had been very brief and superficial for $57 \%$ of the participants. However, a large majority of them (85.2\%) had never had any refresher training on the GCS since they started working. The study also revealed that refresher training on GCS were not associated with knowledge ${ }^{16}$.

Another study found that the majority of the respondents were formally trained or educated in use of the instrument. However, great variation existed in training methods for the use of the instruments, and thus recommend standardized practice in the use of the assessment scales ${ }^{20}$.

\section{Theoretical knowledge}

Theoretical knowledge of the GCS was assessed using five multiple choice questions, each five points. The first question enquired what type of tool the GCS is; whether it is subjective of objective. That question had a score of $12.07 \%$ (48.28\%). This showed that more than half of the nurses $(51.72 \%)$ were not aware that the tool was objective, and not subjective. They need to understand that GCS is indeed an objective and reliable tool that all nurses should become familiar with regardless of their place of employment ${ }^{2-5}$.

The second question was on the best (or highest) score possible for the Scale. $72.41 \%$ of the respondents got it correctly. In one study, $97.6 \%$ got it correct $^{1}$. 
The third question was on the worst (or lowest) score possible for the Scale. Only $41.38 \%$ of the respondents got the correct answer. One study found that more than half (56.3\%) of the participants did not know the lowest score for $\mathrm{GCS}^{14}$.

The fourth question concerned the interval of GCS that indicates critically status of the patient and must be used as an alarming sign by the nurse. $89.66 \%$ of the respondents got it correctly while in one study ${ }^{1}, 80.3 \%$ got it correct. According to one study ${ }^{14}, 78.8 \%$ of the respondents were unable to identify a GCS score that indicates a critical situation the examiner should be aware of while assessing the patients.

The fifth question concerned the interval of GCS that indicates moderate severity. $82.76 \%$ of the respondents got it correct while in one study ${ }^{1}, 81.1 \%$ answered correctly. A different study ${ }^{14}$ found that $62.0 \%$ of the respondents were unable to identify the range of GCS results that indicates a moderate neurological condition.

\section{Practical application of knowledge}

To ensure patient safety, the GCS has to be performed accurately.

Practical application of knowledge about the GCS was assessed using fifteen multiple choice questions, each five points. The questions assessed practical application of knowledge about the GCS in clinical scenarios by grading the different parameters of the GCS. The overall average score for the fifteen questions was 37.76 out of $75(50.35 \%)$.

The component of eye-opening had four questions, and the average score was 10.69 out of 20 $(53.45 \%)$. The component of verbal response had five questions, and the average score was 10.21 out of $25(51.05 \%)$. The component of motor response had six questions and was the worst performed with an average score of 7.95 out of $30(47.70 \%)$.

One study found that GCS motor score performance was the least accurate component ${ }^{22}$. Another study found that $50.67 \%$ of the participants responded correctly to eye opening responses but only $34.67 \%$ responded correctly to the motor response ratings. However, a better accuracy was achieved in the verbal response category with $89.33 \%$ participants responding correctly ${ }^{24}$. The results were consistent with previously published studies demonstrating that the motor response rating is most problematic in relation to rating accuracy. One study has shown there are differences in attitudes and self-confidence in using the GCS between nurses of different demographics, and that there are a variety of factors influencing their attitudes and confidence ${ }^{21}$.

\section{Knowledge of GCS}

Knowledge of GCS was assessed by combining the theoretical knowledge and practical application scores.

Overall results for the study showed that the average score was $54.46 \%$, revealing that the nurses had poor knowledge of the GCS. Unfortunately, many studies that have been conducted 
to assess knowledge of nurses about the GCS have reported similar results showing that nurses generally have poor knowledge about this important tool ${ }^{1,6,8-26}$. One study found that, although the majority of nurses in that study showed a good knowledge about GCS, however, some nurses had poor knowledge about the scale ${ }^{1}$.

Another study revealed that the percentages of nurses who had a good knowledge about GCS was $50.6 \%$ and those who had poor knowledge was $49.4 \%{ }^{12}$. Kimboka ${ }^{14}$ found that only 13.3 $\%$ of participants had high level of knowledge regarding GCS. Another one showed that $41.7 \%$ of respondents had good, $25.2 \%$ moderate and $33.0 \%$ had poor knowledge of the GCS ${ }^{15}$. One study agrees that many studies in different countries have reported low levels of knowledge of the GCS among nurses. In that study, it was found that a little more than half of the participants $(50.4 \%)$ had low knowledge of the GCS as a whole ${ }^{16}$, while yet another study found that 55.56 $\%$ of the nurses had poor knowledge followed by $41.48 \%$ and $2.96 \%$ with satisfactory knowledge and good knowledge, respectively ${ }^{17}$.

As already mentioned previously, knowledge for this study was assessed using 20 multiple choice questions. The first five multiple choice questions tested theoretical knowledge about the GCS, while the remaining fifteen questions assessed practical application of knowledge about the GCS in clinical scenarios by grading the different parameters of the GCS. The overall score for theoretical knowledge on GCS was 16.9 out of 25 while practical knowledge scored 37.76 out of 75 . Out of a possible range of scores from 0 to 20 , respondents had a mean score of 10.93. On theoretical knowledge about the GCS, out of a possible range of scores from 0 to 5 , the respondents had a mean score of 3.38. Concerning practical knowledge related to the application of GCS in clinical scenarios, out of a possible range of scores from 0 to 15 , respondents had a mean score of 7.55. This, therefore, shows that for the questions on the basic theoretical concepts about the GCS, the respondents demonstrated moderate knowledge on these items getting $67.6 \%$ of the answers correctly. However, when it came to questions about applying knowledge of the GCS in clinical scenarios, the respondents demonstrated poor knowledge on these items getting $50.35 \%$ of the questions correctly, showing that although they have knowledge about the basic theoretical concepts of the GCS, they are not able to apply that basic knowledge in clinical scenarios appropriately. This scenario could be attributed to lack of refresher training on GCS when nurses start working.

However, the findings are not unique; they have been demonstrated elsewhere through other studies $^{1,6,8-26}$. For example, the results of one study showed that respondents scored highly $(>75 \%)$ on knowledge questions but poor $(<36 \%)$ to moderate $(<67 \%)$ on questions that concern clinical application of results and GCS use in special situations ${ }^{15}$. Another study found that nurses demonstrated a good theoretical knowledge on clear indication of GCS and its importance, assessment of eye opening and verbal response. However, there were some 
critical points that needed clarification on the aspect of examining a motor response from patients $^{18}$. While another study found that, for the questions on basic theoretical concepts of the GCS, majority of the participants $(62.6 \%)$ demonstrated good knowledge on these items, getting $80 \%$ of the answers correctly. However, when it came to questions about applying knowledge of the GCS in clinical scenarios, only $5.2 \%$ of participants were able to answer 80 $\%$ of the questions correctly. Out of a possible range of scores from 0 to 20 , the participants had a mean score of $11.99^{16}$. These findings suggest that nurses are not able to integrate their theoretical knowledge of the GCS with its practical application in the clinical setting.

The results of several studies illustrate inconsistency and confusion when using the GCS in practice, which has the potential to compromise care. Thus, a standardized approach to education is necessary to ensure greater interrater reliability of assessment not only within nursing, but across professions ${ }^{23-26}$.

\section{CONCLUSION}

The findings from this study show that nurses had low levels of knowledge about the GCS, and that they were not able to apply basic theoretical knowledge to clinical scenarios appropriately. This inadequate knowledge definitely limits their capacity for clinical judgement and decision making in managing unconscious patients.

\section{RECOMMENDATIONS}

1. It is recommended that the length and depth of both the teaching of the theoretical concepts and the skill of performing GCS assessment be reviewed by nursing schools for basic and postbasic educational programmes for nursing students with the view of adapting more comprehensive in-depth teaching combined with demonstrations in classrooms and during clinical experiences in order to enhance nurses' knowledge of GCS.

2. Implementation of regular Continuous Professional Development (CPD), Continuous Medical Education (CME) and refresher training sessions.

\section{ACKNOWLEDGEMENT}

We sincerely appreciate the nurses who agreed to participate in this study as respondents, without whom this study would not have been possible.

\section{REFERENCES}

1. Santos, W. C., Vancini-Campanharo, C.R., Lopes, M. C. B. T., Okuno, M. F. P., \& Batista, R. E. A. (2016). Assessment of nurse's knowledge about Glasgow Coma Scale at a university hospital. Einstein (Sao Paulo), 14(2), 213-218. https://doi.org/10.1590/S1679-45082016AO3618

2. Gaines K. (2019). Understanding the Glasgow Coma Scale. Retrieved from:https://nurse.org/articles/glasgow-coma-scale/ 
3. Yousuf, K. (2019). Glasgow Coma Scale (GCS). Retrieved from: https://geekymedics.com/glasgow-coma-scale-gcs/

4. TraumaticBrainInjury.com. (2019). Glasgow Coma Scale. Retrieved from:https://www.traumaticbraininjury.com/glasgow-coma-scale/

5. Glasgowcomascale.org. (2019). The Glasgow structured approach to assessment of the Glasgow Coma Scale. Retrieved from: https://www.glasgowcomascale.org/what-isgcs/

6. Mattar, I., Liaw, S. Y., Chan, M. F. (2013). A study to explore nurses' knowledge in using the Glasgow Coma Scale in an acute care hospital. The Journal of Neuroscience Nursing, 45(5), 272-80. https://doi.org/10.1097/JNN.0b013e31829db970

7. Liverpool Health Service. (2019). Neurological assessment using the Glasgow Coma $\begin{array}{llll}\text { Scale } & (G C S) \text { from: }\end{array}$ https://www.mccc.edu/nursing/documents/glasgowcomascale.pdf

8. Summers C, McLeod A. (2017). What influences nurses to undertake accurate assessment of the Glasgow Coma Scale? British Journal of Neuroscience, 13(1). https://doi.org/10.12968/bjnn.2017.13.1.24

9. Ahamed, S. T., \& Ebraheim, M. N. (2017). Glasgow Coma Scale technique: Effect of theoretical and practical educational program on nurses' compliance. Journal of Nursing and Health Science, 6(3), 21-28.

10. Jaddoua, B. A., Mohammed, W. A., \& Abbas, A. D. (2013). Assessment of nurse's knowledge concerning Glasgow Coma Scale in neuro surgical wards. Journal of Kufa for Nursing Science, 3(2). https://www.iasj.net/iasj?func=fulltext\&aId=79447

11. Teles, M., Bhupali, P., \& Madhale, M. (2013). Effectiveness of self instructional module on knowledge and skills regarding use of Glasgow Coma Scale in neurological assessment of patients among nurses working in critical care units of KLE Dr. Prabhakar Kore Hospital and Medical Research Centre, Belgaum. Journal of Krishna Institute of Medical Sciences University, 2(1). http://www.jkimsu.com/jkimsu-vol2 no1 / jkimsu, \% 20 vol \% 202, \%20 no \% 201, \%20 jan \%20-\%20june\%202013,\%2098104.pdf

12. Ayoub, A. Y., Saifan, A., Alaween, M., Almansouri, E. S., Hussain, H. Y., \& Salim, N. A. (2018). Assessment of nurse's knowledge about Glasgow Coma Scale at al Dhafra hospitals. Journal of Clinical Review \& Case Reports, 3(7). https://doi.org/10.4172/2573-4571-C6-029

13. Al-Quraan, H. A., \& AbuRuz, M. E. (2016). Assessment of Jordanian nurses' knowledge to perform Glasgow Coma Scale. European Scientific Journal, 12(27). http://dx.doi.org/10.19044/esj.2016.v12n27p208 
14. Kimboka, J. J. (2017). Nurses' knowledge and practice in the application of the Glasgow Coma Scale in the intensive care units and emergency department at Muhimbili National Hospital and Muhimbili Orthopaedic Institute, in Dar Es Salaam.http://dspace.muhas.ac.tz:8080/xmlui/bitstream/handle/123456789/2180/Kim boka\%2C\%202017.pdf? sequence $=1 \&$ isAllowed $=\mathrm{y}$

15. Ehwarieme, T. A., \& Anarado, A. N. (2016). Nurses' knowledge of Glasgow Coma Scale in neurological assessment of patients in a selected tertiary hospital in Edo State, Nigeria. Africa Journal of Nursing and Midwifery, 18(2), 74-86. https://doi.org/10.25159/2520-5293/1068

16. Alhassan, A., Fuseini, A., \& Musah, A. (2019). Knowledge of the Glasgow Coma Scale among nurses in a tertiary hospital in Ghana. Nursing Research and Practice, Article 5829028. https://doi.org/10.1155/2019/5829028

17. Singh, H. K. B., Chong, M. C., Thambinayagam, H. C., bin Zakaria, M. I., Cheng, S. T., Tang, L. Y., \& Azahar, N. H. (2016). Assessing nurses knowledge of Glasgow Coma Scale in emergency and outpatient department. Nursing Research and Practice, Article 8056350, 1-5. https://doi.org/10.1155/2016/8056350

18. Catangui, E. (2019). Improving Glasgow Coma Scale (GCS) competency of nurses in one acute stroke unit: A nursing initiative project. Journal of Nursing and Practice, 3(1). https://doi.org/10.36959/545/370

19. Waterhouse, C. (2013). An audit of nurses' conduct and recording of observations using the Glasgow Coma Scale. British Journal of Neuroscience Nursing, 4(10). https://doi.org/10.12968/bjnn.2008.4.10.31343

20. Vink, P., Tulek, Z., Gillis, K., Jönsson, A., Buhagiar, J., Waterhouse, C., \& Poulsen, I. (2018). Consciousness Assessment: A Questionnaire of Current Neuroscience Nursing Practice in Europe. Journal of Clinical Nursing, 27(21-22), 3913-3919. https://doi.org/10.1111/jocn.14614

21. Mattar, I, Liaw, S.Y., Chan, M. F. (2015). Nurses' self-confidence and attitudes in using the Glasgow Coma Scale: A primary study. Nursing in Critical Care, 20(2), 98-107. https://doi.org/10.1111/nicc.12077

22. Enriquez, C.M., Chisholm, K. H., Madden, L. K., Larsen, A. D., de Longpré, T., \& Stannard, D. (2019). Glasgow Coma Scale: Generating clinical standards. Journal of Neuroscience Nursing, $51(3)$ $142-$ 146. https://doi.org/10.1097/JNN.0000000000000448

23. Cook, N. F., Braine, M. E., Trout, R. (2019). Nurses' understanding and experience of applying painful stimuli when assessing components of the Glasgow Coma Scale. Journal of Critical Nursing. https://doi.org/10.1111/jocn.15011 
24. Heron, R., Davie, A., Gillies, R., \& Courtney, M. (2001). Interrater reliability of the Glasgow Coma Scale scoring among nurses in sub-specialties of critical care. Australian Critical Care, 14(3), 100-105. https://doi.org/10.1016/s10367314(01)80026-6

25. Hussein, H. A. (2015). Effect of instructional guidelines about coma and Pediatric Glasgow Coma Scale on knowledge and skills of Pediatric intensive care nurses. World Journal of Nursing $\quad$ Sciences, $1(1), \quad$ 12. https://doi.org/10.5829/idosi.wjns.2015.1.1.93200

26. Shehab, M. S., Ibrahim, N. M., \& Abd-Elkader, H. (2018). Impact of an educational program on nurses' knowledge and practice regarding care of traumatic brain injury patients at intensive care unit at Suez Canal University Hospital. International Journal of Caring Sciences, 11(2), 1104-1116.

\section{BJMHR is \\ - Peer reviewed \\ - Monthly \\ - Rapid publication \\ - Submit your next manuscript at}


